\title{
LETTER
}

Stem cell biology

\section{Maternal exposure to fine particulate matter during pregnancy induces progressive senescence of hematopoietic stem cells under preferential impairment of the bone marrow microenvironment and aids development of myeloproliferative disease}

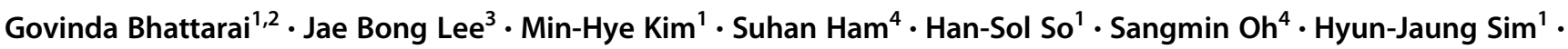 \\ Jeong-Chae Lee ${ }^{1,2} \cdot$ Mijung Song $^{4} \cdot$ Sung-Ho Kook ${ }^{1}$
}

Received: 4 July 2019 / Revised: 2 October 2019 / Accepted: 17 November 2019 / Published online: 27 November 2019

(c) The Author(s) 2019. This article is published with open access

\section{To the Editor:}

During the last few decades, industrial development and expansion have led to significantly increased levels of fine particulate matter (PM) in the air, including particles with aerodynamic diameters smaller than $2.5 \mu \mathrm{m}$ (referred to as $\mathrm{PM}_{2.5}$ ) [1]. $\mathrm{PM}_{2.5}$ can have negative effects on air quality and threatens human health. Furthermore, it can have effects on tissue-specific stem progenitor cells [2, 3]. However, little is known about the mechanisms underlying the effects of $\mathrm{PM}_{2.5}$ on stem progenitor cells, particularly hematopoietic stem progenitor cells (HSPCs). There is growing evidence that maternal exposure to $\mathrm{PM}_{2.5}$ during pregnancy

These authors contributed equally: Govinda Bhattarai, Jae Bong Lee, Min-Hye Kim

Supplementary information The online version of this article (https:// doi.org/10.1038/s41375-019-0665-8) contains supplementary material, which is available to authorized users.

\section{Mijung Song}

mijung.song@jbnu.ac.kr

$\bowtie$ Sung-Ho Kook

kooksh@jbnu.ac.kr

1 Department of Bioactive Material Sciences, Research Center of Bioactive Materials, Chonbuk National University, Jeonju 54896, South Korea

2 Institute of Oral Bioscience and School of Dentistry, Chonbuk National University, Jeonju 54896, South Korea

3 Thermal Hydraulics and Severe Accident Research Division, Korea Atomic Energy Research Institute, Deajeon 34057, South Korea

4 Department of Earth and Environmental Sciences, Chonbuk National University, Jeonju 54896, South Korea can harm both the embryo and progeny [4-6]. The embryo and fetus are more susceptible to external stress than the adult. Although there is growing evidence regarding the detrimental risks to the embryo and offspring that have been maternally exposed to $\mathrm{PM}_{2.5}$ during pregnancy [4-6], little is known about the effects of maternal $\mathrm{PM}_{2.5}$ exposure to stem cells, which begin to emerge, activate, and mature during embryo development.

In this study, we developed an atmospheric simulation chamber (ASC) (Supplementary Fig. 1a), and demonstrated that maternal exposure to $\mathrm{PM}_{2.5}(2 \mathrm{~h}$ on five consecutive days, $\sim 50 \mu \mathrm{g} / \mathrm{m}^{3} \mathrm{PM}_{2.5}$ mass concentration, Supplementary Tables 1 and 2, Supplementary Fig. 1b, c) during pregnancy can affect the lungs of the fetus. The $\mathrm{PM}_{2.5}$ that reached the fetal lungs incurred oxidative stress and inflammation, in agreement with previous reports (Supplementary Fig. 1d) $[7,8]$. The initially detrimental phenomena in the fetal lungs that were triggered by maternal exposure of $\mathrm{PM}_{2.5}$ continued after birth, leading to impairment in the bronchiole structure of the offspring born to the $\mathrm{PM}_{2.5}$-exposed dam (Supplementary Fig. 1e). A greater number of cells, including $\mathrm{CD}^{+}$and $\mathrm{CD}^{+}{ }^{+} \mathrm{T}$ cells, infiltrated the bronchioles of $\mathrm{PM}_{2.5}$-exposed offspring, which also exhibited mild fibrosis (Supplementary Fig. 1f, g). The NLRP3 inflammasome, one of the most fully characterized inflammasomes, has been implicated in the pathogenesis of inflammatory and fibrosis diseases. A previous study confirmed that $\mathrm{PM}_{2.5}$ could activate the NLRP3 inflammasome [9]. Indeed, we verified the upregulation of NLRP3 in the lung tissues of $\mathrm{PM}_{2.5}$-exposed offspring. The results further confirmed that $\mathrm{PM}_{2.5}$ promoted the activation of cleaved caspase-1 and IL-1 $\beta$, which are representative markers of inflammasome activation (Supplementary Fig. 1h, i). Besides the lung, oxidative stress and inflammation were also observed in other tissues (Supplementary Fig. 2a) such 
as the liver, brain, kidney, spleen, and thymus of the $\mathrm{PM}_{2.5^{-}}$ exposed offspring. However, there was no severe inflammation and oxidative stress in the fetus liver (FL) and brain and also no alteration in bone development in the fetus (Supplementary Fig. 2a-c). These results indicate that maternal exposure to fine $\mathrm{PM}_{2.5}$ during pregnancy can also detrimentally affect other tissues of the offspring by inducing systemic inflammation, but not the fetus.

However, maternal exposure to $\mathrm{PM}_{2.5}$ did not directly affect FL hematopoietic stem cell (HSCs), which serves as a main site for the expansion and differentiation of HSCs during fetal life, until hematopoiesis shifts to the bone marrow (BM) around birth (Supplementary Fig. 3a, b). No detrimental effects of maternal $\mathrm{PM}_{2.5}$ in FL HSPCs carried over to the BM HSPCs in offspring of 2 months, which were born to $\mathrm{PM}_{2.5}$-exposed pregnant dam (Supplementary Fig. 3c-f). Of note, $\mathrm{PM}_{2.5}$-exposed offspring of 6 months exhibited senescent phenotypes in BM HSCs, as evidenced by lower clonogenic formation, donor cell-derived reconstitution, and self-renewal, as well as higher levels of mitochondrial ROS, Nrf2 expression, p38 phosphorylation, SA- $\beta$-gal activity, and biased-myeloid differentiation (Fig. 1a-f and Supplementary Fig. 4a-h) [10, 11]. mRNA levels of cyclin-dependent kinase inhibitors such as p16, $p 21, p 19$, and $p 15$ also increased in BM HSCs of $\mathrm{PM}_{2.5^{-}}$ exposed offspring compared with those of control offspring
(Supplementary Fig. 4i). $\mathrm{PM}_{2.5}$-exposed offspring-derived BM HSCs exhibited higher levels of DNA double-strand breaks compared with their control offspring-derived counterparts, as demonstrated by flow cytometry using a $\gamma$-H2AX antibody (Fig. 1g). However, there were no significant differences in the numbers of circulating WBCs, RBCs, and platelets between control and $\mathrm{PM}_{2.5}$-exposed offspring (Supplementary Fig. 4j). These results led us to hypothesize that, although HSCs are not directly affected by maternal exposure to $\mathrm{PM}_{2.5}$ during pregnancy, they may be progressively altered to be senescent under the abnormal BM microenvironment formed via non-cell autonomous processes. As expected, the exposure to fine $\mathrm{PM}_{2.5}$ during pregnancy impaired the $\mathrm{BM}$ microenvironment by inducing ROS-mediated senescence of bone cells and MSCs in offspring of 2 months (Supplementary Fig. 5). A previous report indicated that age-associated bone cell senescence enhances osteoclastogenesis [12]. Our findings were consistent with these results, as $\mathrm{PM}_{2.5}$-exposed offspring of 2 months exhibited a high level of osteoclast activity, supported by relatively increased RANKL to OPG levels and high levels of inflammatory cytokines (Supplementary Fig. 6a-g). An age-related BM microenvironment with preferential osteogenesis and senescent osteoblastic cells in the $\mathrm{PM}_{2.5}$-exposed offspring generated high levels of proteolytic enzymes such as MMP-2, MMP-9, and CTK, which a
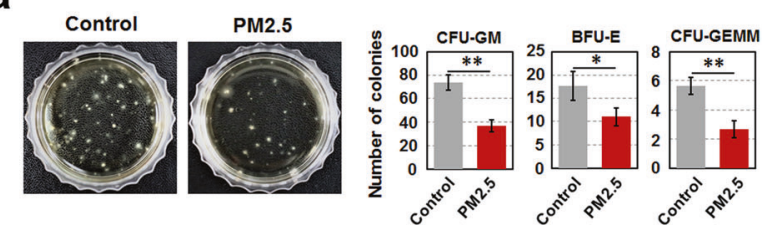

C
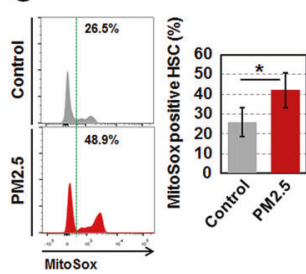

d

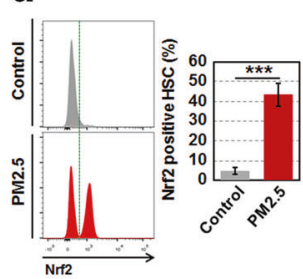

e

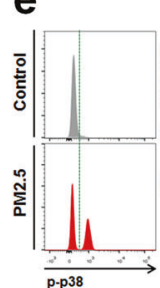

Fig. 1 Maternal exposure to fine $\mathrm{PM}_{2.5}$ during pregnancy leads to the progressive senescence of HSCs in offspring of 6 months. a For the colony assay, BM cells $\left(2 \times 10^{4}\right.$ per dish $)$ in $\mathrm{PM}_{2.5}$-exposed offspring of 6 months were incubated in a methylcellulose-based medium for 12 days and the colonies formed were counted. Representative data are shown for three independent experiments. b For long-term competitive repopulating activity, equal numbers $\left(5 \times 10^{5}\right)$ of $\mathrm{BM}$ cells from control or $\mathrm{PM}_{2.5}$-exposed offspring were co-transplanted with those from competitor mice (CD45.1) into lethally irradiated recipient mice $(\mathrm{CD} 45.1 / 2,1000 \mathrm{rads}, n=7)$ that were also transplanted with BM cells $\left(1 \times 10^{6}\right)$ of CD45.1/2 mice after the first transplantation. PB was collected from the recipient mice at 4 months post-transplantation and the ratio of CD45.1/CD45.2 was assessed by flow cytometry. Donor-
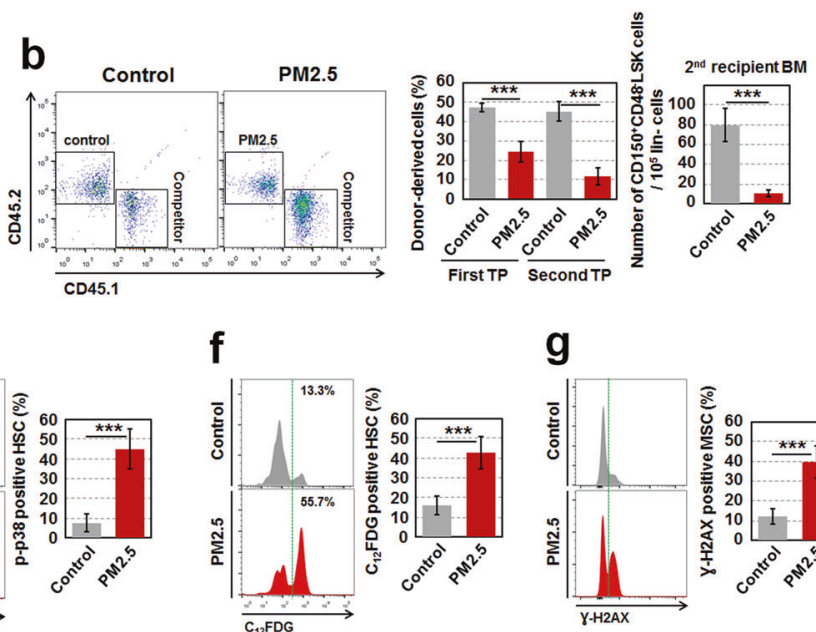

f
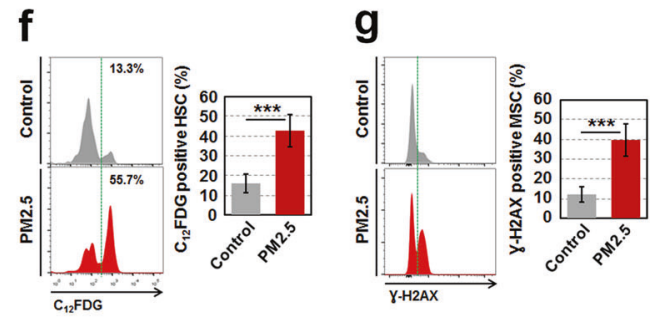

derived HSCs engrafted in the BM of the secondary recipient mice were measured by flow cytometry after the procedure of lineage cell depletion $(n=4)$. c Levels of mitochondrial superoxide anions in the BM HSCs of offspring of 6 months were measured with MitoSOX ${ }^{\mathrm{TM}}$ Red reagent using flow cytometry $(n=11)$. Levels of Nrf2 $(\mathbf{d})$ and p38 phosphorylation (e) were analyzed in the BM HSCs of the offspring after the fixation and permeabilzation procedure $(n=7)$. f SA- $\beta$-gal activity in BM HSCs of the offspring were measured using incubating the cells with $\mathrm{C}_{12} \mathrm{FDG}$, a $\beta$-galactosidase substrate $(n=11)$. g Levels of $\gamma-\mathrm{H} 2 \mathrm{AX}$ were analyzed in the BM HSCs of the offspring after the fixation and permeabilization procedure $(n=5)$. All data are presented as the means $\pm \mathrm{SD}$. $* p<0.05$, **p $<0.01$, and $* * * p<0.001$ vs. control, as determined by Student's $t$ tests 


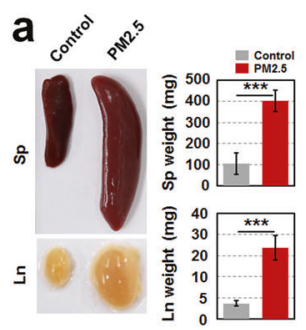

b

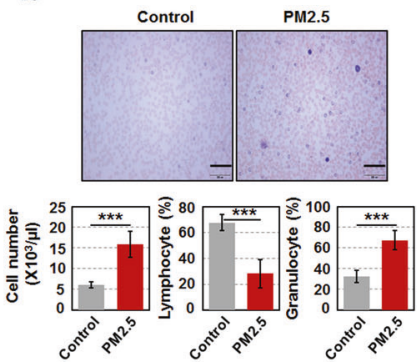

C

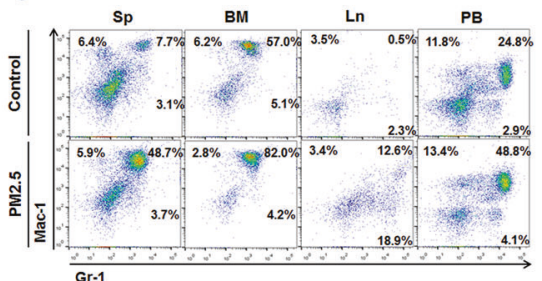

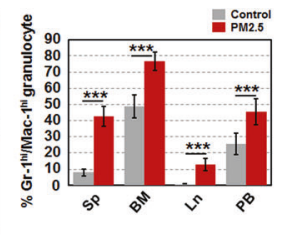

(1)

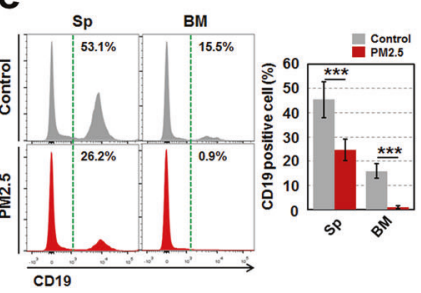

Fig. 2 Maternal $\mathrm{PM}_{2.5}$-exposed offspring have the potential to develop a myeloproliferative disease. a Photographs of the Sp and Ln from control and maternal $\mathrm{PM}_{2.5}$-exposed offspring at 1 year of age and weights of those were measured $(n=4)$. b May-Grünwald-Giemsa stained blood smear (upper panels) and leukocyte number and the proportion of lymphocytes and granulocytes (lower panels) from the old offspring $(n=5)$. Scale bars are $200 \mu \mathrm{m}$. c Percentage of $\mathrm{Gr}-1^{+} / \mathrm{Mac}-1^{+}$granulocytes in $\mathrm{Sp}, \mathrm{BM}, \mathrm{Ln}$, and $\mathrm{PB}$ of the old offspring $(n=5)$. d Myeloperoxidase immunohistochemistry was

are involved in the regulation of HPC retention in the BM (Supplementary Fig. 6h). The preferential impairment of the $\mathrm{BM}$ microenvironment in $\mathrm{PM}_{2.5}$-exposed offspring led to HSC senescence in non-cell autonomous mechanisms, as evidenced by the induction in senescence and functional defects of donor cell-derived HSCs that were transplanted into conditioned $\mathrm{PM}_{2.5}$-exposed offspring recipients (Supplementary Fig. 7). Taken together, these findings demonstrate that maternal exposure to $\mathrm{PM}_{2.5}$ during pregnancy preferentially impairs the BM microenvironment, causing it to be aged, after which BM HSCs progressively undergo senescence via non-cell autonomous mechanisms.

The modulating effects of maternal $\mathrm{PM}_{2.5}$ exposure on BM microenvironment-mediated HSCs were different from those observed when adolescent mice (4 weeks of age) were exposed to $\mathrm{PM}_{2.5}$, as the $\mathrm{BM}$ microenvironment and $\mathrm{BM}$ HSCs were hardly affected in adolescent $\mathrm{PM}_{2.5}$-exposed mice (Supplementary Fig. 8). These results illustrate that $\mathrm{PM}_{2.5}$ has different impacts on mice depending on the time at which they are exposed and displays more detrimental effects during the embryo stage than in the adult.

Similar to a previous study that showed an aged microenvironment can contribute to aging-related myeloproliferative disease [13], our current findings demonstrated that a total of 9 out of $25(\sim 36 \%) \mathrm{PM}_{2.5}$-exposed 1-year-old offspring had the potential to develop a myeloproliferative disease along with increased SA- $\beta$-gal activity in MSCs and HSCs during conducted in hematopoietic ( $\mathrm{Ln}$ and $\mathrm{Sp}$ ) and nonhemtopoietic organs (lung and liver) of the old offspring to measure infiltrated blast cells to the organs. A representative result is shown $(n=5)$. Scale bars are 100 (left) and 20 (right) $\mu \mathrm{m}$. DAB-positive cell intensity was measured by ImageJ-win64. e Percentage of B cells $\left(\mathrm{CD} 19^{+}\right.$cells) in Sp and BM of the old offspring $(n=5)$. All data are presented as the means \pm SD. $* * p<0.01$ and $* * * p<0.001$ vs. control, as determined by Student's $t$ tests

aging (Supplementary Fig. 9), as evidenced by the following: massive enlargement of the spleen (Sp) and lymph node (Ln); increased BM and Sp cellularity; increased number of circulating leukocytes; increased percentage of $\mathrm{Gr}-1^{+} / \mathrm{Mac}-1^{+}$ granulocytes in the $\mathrm{Sp}, \mathrm{BM}, \mathrm{Ln}$, and $\mathrm{PB}$; higher percentage of immature myeloid cells, such as c-Kit ${ }^{+} \mathrm{Gr}-1^{+}$and c-Kit ${ }^{-} \mathrm{Gr}-1^{+}$ cells; lower percentage of mature myeloid cells, such as c-Kit ${ }^{+} \mathrm{Gr}-1^{+} \mathrm{Ly}_{6 \mathrm{G}}{ }^{+}$and $\mathrm{c}-\mathrm{Kit}^{-} \mathrm{Gr}-1^{+} \mathrm{Ly} 6 \mathrm{G}^{+}$cells; and higher infiltration of myeloperoxidase-stained massive blast cells in the Ln, Sp, and nonhematopoietic organs, such as the lungs and liver in $\mathrm{PM}_{2.5}$-exposed old offspring (Fig. 2 and Supplementary Fig. 10).

It has been extensively demonstrated that the inductive generation of oxidative stress by $\mathrm{PM}_{2.5}$ exposure is a major determinant in triggering tissue impairment. In this study, we detected increased oxidative stress in the lungs of E16.5, which was immediately observed after maternal $\mathrm{PM}_{2.5}$ exposure for five consecutive days. The administration of drinking water that contained NAC to pregnant mice during $\mathrm{PM}_{2.5}$ exposure limited the generation of oxidative stress and inflammasomes in the lungs and ameliorated the $\mathrm{PM}_{2.5^{-}}$ induced impairment of the BM microenvironment, followed by the prevention of BM HSC senescence and normal clonogenic formation (Supplementary Fig. 11). Despite the preventive effects of NAC treatment in $\mathrm{PM}_{2.5}$-exposed pregnant mice, the offspring born to those with NAC underwent growth retardation and had a low body weight 
(data not shown). As a previous report indicated that a slight change in ROS levels is closely involved in various cell signaling processes and can directly affect embryonic development [14], the application of NAC to prevent ROSmediated detrimental effects in $\mathrm{PM}_{2.5}$-exposed mice should be considered during the embryo stage to avert any unexpected additional damage.

The increased detrimental effects of $\mathrm{PM}_{2.5}$ exposure to the embryo rather than adolescent mice via the modulation of the BM microenvironment and HSCs, as demonstrated in our findings (Fig. 1 and Supplementary Figs. 3-8) may have been due to an incomplete defense system in the embryo that is unable to prevent $\mathrm{PM}_{2.5}$-induced oxidative stress.

In summary, maternal exposure to fine $\mathrm{PM}_{2.5}$ during pregnancy destructively affects the fetal lungs by inducing oxidative stress and these harmful effects last to adulthood, followed by the induction of inflammasomes and impaired bronchioles. In addition, maternal exposure of $\mathrm{PM}_{2.5}$ causes the progressive senescence of HSCs via the ROS-p38 MAPK and Nrf2 pathway under the exposure-induced preferential impairment of the BM microenvironment with agerelated phenotypes. Our results demonstrate that the embryo is more vulnerable to $\mathrm{PM}_{2.5}$ exposure than the adolescent, particularly in relation to the BM microenvironmentassociated modulation of HSCs. However, only oxidative stress inhibitor experiments have been performed in the present study and show the evidence that the effects of $\mathrm{PM}_{2.5}$ were mediated by activation of oxidative stress signaling. On the other hand, further research is needed to investigate the exact role and mechanism of other signaling molecules in the effects of $\mathrm{PM}_{2.5}$ by using gene knockout or inhibitor experiments.

\begin{abstract}
Acknowledgements The authors wish to thank Joshua Lee for scientific editing of the manuscript. For technical support, we thank Gwanggon Jo. This work was supported by the Basic Science Research Program through the National Research Foundation of Korea (NRF) grant funded by the Ministry of Science, Information and Communications Technology and Future Planning, Korea (2017R1A2B4009269 and 2019R1A2C 2084453) and the Ministry of Education (2017R1A6A3A11028201 and 2018R1D1A1B07047162). This work was supported by the National Research Foundation of Korea (NRF) grant funded by the Korea government (Ministry of Science and ICT) (NRF-2017M2A8A4015028) and also supported by Korea Institute of Toxicology (KK-1905-02).
\end{abstract}

Author contributions JBL, MS, and SHK conceived and designed the experiments. GB, JBL, MHK, SH, HSS, SO, HJS, MS, and SHK performed the experiments and analyzed the data. JCL, MS, and SHK contributed reagents, materials, and analytical tools. GB, JBL, MHK, MS, and SHK wrote the manuscript and JBL, MS, and SHK edited the manuscript.

\section{Compliance with ethical standards}

Conflict of interest The authors declare that they have no conflict of interest.
Publisher's note Springer Nature remains neutral with regard to jurisdictional claims in published maps and institutional affiliations.

Open Access This article is licensed under a Creative Commons Attribution 4.0 International License, which permits use, sharing, adaptation, distribution and reproduction in any medium or format, as long as you give appropriate credit to the original author(s) and the source, provide a link to the Creative Commons license, and indicate if changes were made. The images or other third party material in this article are included in the article's Creative Commons license, unless indicated otherwise in a credit line to the material. If material is not included in the article's Creative Commons license and your intended use is not permitted by statutory regulation or exceeds the permitted use, you will need to obtain permission directly from the copyright holder. To view a copy of this license, visit http://creativecommons. org/licenses/by/4.0/.

\section{References}

1. Monks PS, Granier C, Fuzzi S, Stohl A, Williams ML, Akimoto $\mathrm{H}$, et al. Atmospheric composition change-global and regional air quality. Atmos Environ. 2009;43:5268-350.

2. Abu-Elmagd M, Alghamdi MA, Shamy M, Khoder MI, Costa M, Assidi $\mathrm{M}$, et al. Evaluation of the effects of airborne particulate matter on bone marrow-mesenchymal stem cells (BM-MSCs): cellular, molecular and systems biological approaches. Int J Environ Res Public Health. 2017. https://doi.org/10.3390/ijerph14040440.

3. Cui Y, Sun Q, Liu Z. Ambient particulate matter exposure and cardiovascular diseases: a focus on progenitor and stem cells. $\mathrm{J}$ Cell Mol Med. 2016;20:782-93.

4. Twum C, Zhu J, Wei Y. Maternal exposure to ambient PM2.5 and term low birthweight in the state of Georgia. Int J Environ Health Res. 2016;26:92-100.

5. Zhang T, Zheng X, Wang X, Zhao H, Wang T, Zhang H. Maternal exposure to $\mathrm{PM}_{2.5}$ during pregnancy induces impaired development of cerebral cortex in mice offspring. Int $\mathrm{J}$ Mol Sci. 2018;19:257-73.

6. Zhou Y, Gilboa SM, Herdt ML, Lupo PJ, Flanders WD, Liu Y. Maternal exposure to ozone and $\mathrm{PM}_{2.5}$ and the prevalence of orofacial clefts in four U.S. states. Environ Res. 2017;153: $35-40$.

7. Deng X, Zhang F, Rui W, Long F, Wang L, Feng Z, et al. PM2.5induced oxidative stress triggers autophagy in human lung epithelial A549 cells. Toxicol Vitr. 2013;27:1762-70.

8. Hong Z, Guo Z, Zhang R, Xu J, Dong W, Zhuang G, et al. Airborne fine particulate matter induces oxidative stress and inflammation in human nasal epithelial cells. Tohoku J Exp Med. 2016;239:117-25.

9. He Y, Hara H, Nunez G. Mechanism and regulation of NLRP3 inflammasome activation. Trends Biochem Sci. 2016;41:1012-21.

10. Kook SH, Yun CY, Sim HJ, Bhattarai G, Lee BC, Lee KY, et al. Smad4 in osteoblasts exerts a differential impact on HSC fate depending on osteoblast maturation stage. Leukemia. 2016;30: 2039-46.

11. Rossi DJ, Bryder D, Weissman IL. Hematopoietic stem cell aging: mechanism and consequence. Exp Gerontol. 2007;42: 385-90.

12. Marie PJ. Bone cell senescence: mechanisms and perspectives. J Bone Min Res. 2014;29:1311-21.

13. Vas V, Wandhoff C, Dörr K, Niebel A, Geiger H. Contribution of an aged microenvironment to aging-associated myeloproliferative disease. PLoS One. 2012;7:E31523.

14. Dennery PA. Effects of oxidative stress on embryonic development. Birth Defects Res C Embryo Today. 2007;81:155-62. 